\title{
Brain Region Specificity of Mitochondrial Biogenesis and Bioenergetics Response to Nrf2 Knockdown: A Comparison Among Hippocampus, Prefrontal Cortex and Amygdala of Male Rat Brain
}

\author{
Solmaz Khalifeh ${ }^{1,2 *}$, Fariba Khodagholi ${ }^{3,4}$, Fatemeh Shaerzadeh ${ }^{5}$, Hamed Ghazvini ${ }^{6,7}$, \\ Mohammad-Reza Zarrindast ${ }^{2,8,9}$, Vahid Azizi ${ }^{10}$. \\ ${ }^{1}$ Cognitive and Neuroscience research Center (CNRC) and School of Advanced Sciences in Medicine, Islamic Azad \\ University, Tehran Medical Sciences Branch, Tehran, Iran; ${ }^{2}$ Medical Genomics Research Center and School of \\ Advanced Sciences in Medicine, Tehran Medical Sciences Branch, Islamic Azad University, Tehran, Iran; \\ ${ }^{3}$ Neurobiology Research Center, Shahid Beheshti University of Medical Sciences, Tehran, Iran; ${ }^{4}$ Neuroscience \\ Research Center, Shahid Beheshti University of Medical Sciences, Tehran, Iran; ${ }^{5}$ Molecular Medicine Research \\ Center, Hormozgan Health institute, Hormozgan University of Medical Sciences, Bandar Abbas, Iran; 6 \\ Immunogenetics Research Center, Mazandaran University of Medical Sciences, Sari, Iran; 7 Psychiatry and \\ Behavioral Sciences Research Center, Mazandaran University of Medical Sciences, Sari, Iran; ${ }^{8}$ Department of \\ Pharmacology, School of Medicine, Tehran University of Medical Sciences, Tehran, Iran; 9 Institute for Cognitive \\ Science Studies (ICSS), Tehran, Iran; ${ }^{10}$ Department of Animal Biology, Faculty of Biological Science, Shahid \\ Beheshti University. Tehran, Iran
}

\begin{abstract}
Nuclear factor (erythroid-derived 2)-like 2 (Nrf2) has been identified as the well-known coordinator of intracellular antioxidant defense system. Herein, we aimed to evaluate the effects of Nrf2 silencing on mitochondrial biogenesis

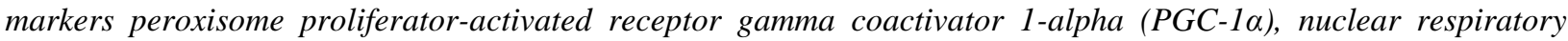
factor-1(NRF-1), mitochondrial transcription factor A (TFAM) and cytochrome $c$ as well activities of two enzymes citrate synthase $(C S)$ and malate dehydrogenase $(M D H)$ in three brain regions hippocampus, amygdala, and prefrontal cortex of male Wistar rats. Small interfering RNA (siRNA) targeting Nrf2 was injected in dorsal third ventricle. Next, western blot analysis and biochemical assays were used to evaluation of protein level of mitochondrial biogenesis factors and CS and MDH enzymes activity, respectively. Based on findings, whilst Nrf2silencing led to notably reduction in protein level of mitochondrial biogenesis upstream PGC-1 $\alpha$ in three brain regions compared to the control rats, the level of NRF-1, TFAM and cytochrome c remained unchanged. Furthermore, although Nrf2 silencing increased CS activity, activity of MDH significantly decreased in hippocampus and prefrontal cortex areas. Interestingly, CS and MDH activities in amygdala did not change after Nrf2 knockdown. In conclusion, the present findings highlighted complexity of interaction of Nrf2 and mitochondrial functions in a brain region-specific manner. However, by outlining the exact interaction between Nrf2 and mitochondria, it would be possible to find a new therapeutic strategies for neurological disorders related to oxidative stress.
\end{abstract}

Key words: Nrf2, Antioxidant defense system, Citrate synthase, Malate dehydrogenase, PGC1- $\alpha$, TFAM

*Author for correspondence: s.khalifeh@iautmu.ac.ir 


\section{INTRODUCTION}

Based on the crucial role in energy production and homeostasis, mitochondria are key players in neuronal cell functions (1). Due to high energy demand and low glycolytic capacity, neuronal cells are extremely dependent on their mitochondria and energy production. Accordingly, precise and coordinated control mechanisms adjust mitochondrial number, distribution, and function through a set of complex processes including mitochondrial fission and fusion, mitophagy and biogenesis (2, $3)$.

Mitochondrial biogenesis, generation of new mitochondria, which requires exquisite coordination of both nucleus and mitochondrial genome can be promoted by exercise, cold, oxidative stress, etc. (4). As the master regulator of mitochondrial biogenesis, transcriptional coactivator peroxisome proliferator-activated receptor- $\gamma$ coactivator-1 $\alpha$ (PGC-1 $\alpha$ ) orchestrates variety of transcription factors including nuclear respiratory factor-1 (NRF-1) and NRF-2 and also mitochondrial transcription factor A (TFAM) (5-7).

Mitochondria play multiple roles in oxido-redox state of cells by balancing detoxification and production of reactive oxygen species (ROS). In addition to intracellular organelles, it has been evidenced that nuclear factor (erythroid-derived 2)-like 1 and 2 (Nrf1 and Nrf2) belonging to the NF-E2 basic-leucine zipper family of proteins which coordinate antioxidant response to oxidative stress $(8,9)$. Nrf2 as a transcription factor interacts with the antioxidant response element (ARE) in the upstream promotor region of many antioxidative genes leading to the promotion of the expression of detoxifying enzymes (10-12). Recently, we reported that Nrf1 knockdown using a specific short interfering RNA molecule (siRNA) leads to alteration in protein level of mitochondrial biogenesis factors in hippocampus, amygdala, and prefrontal cortex of male rats (13). Furthermore, we showed that Nrf2 knockdown induces anxiety-like behavior along with mitochondrial dysfunction and neuronal apoptosis in hippocampus, amygdala, and prefrontal cortex. However, the direct effect of $\mathrm{Nrf} 2$ silencing on mitochondrial biogenesis has remained unknown.

Considering the fact that brain tissue function entirely depends on energy metabolism, tricarboxylic acid cycle (TCA) including eight pivotal enzymes could display rate changes of neuronal cells metabolism. Based on the given roles of mitochondria in glucose metabolism and modulatory function of $\mathrm{Nrf2}$, present study was designed to figure out the effects of Nrf2 knockdown on protein level of mitochondrial biogenesis markers PGC- $1 \alpha$, NRF1, and TFAM along with the activities of TCA enzymes citrate synthase (CS) and malate dehydrogenase (MDH).

\section{MATERIALS AND METHODS}

\section{ANIMALS}

The experiments were carried out on male albino rats of Wistar strain weighing between 200 and $250 \mathrm{~g}$ at the beginning of the surgery. Animals were caged (four per cage) with free access to food and water and kept under constant environmental conditions (12 h-light-dark cycle with lights on from 07:00 to 19:00, at 20-22 ${ }^{\circ} \mathrm{C}$ room temperature). All experimental protocols and treatments were conducted in accordance with the Ethics Committee of Shahid Beheshti University of Medical Sciences.

\section{EXPERIMENTAL DESIGN}

Animals were randomly divided into 3 groups based on injection into dorsal third ventricle $(\mathrm{D} 3 \mathrm{~V})(\mathrm{n}=8)$. The experimental groups were as follows: control group 1 
Brain regions and mitochondrial biogenesis

received $5 \mu$ scrambled siRNA (this siRNA had no homology to any known mammalian gene); control group 2 received $5 \mu \mathrm{l}$ RNase-free water and third group animals received $5 \mu \mathrm{l} \mathrm{Nrf2-siRNA} \mathrm{(5} \mathrm{nmol} \mathrm{siRNA/200} \mu$ l RNAase-free water). Each group was assigned to two subgroups based on the time of decapitation (4 and $8 \mathrm{~h}$ after injection). Silencer select predesigned siRNA specific to Nrf2 was $5^{\prime}$ GCUGAA CUCCUUAGACUCAtt-3' (ID: s136127) (Ambion, Austin, TX, USA) and scrambled siRNA was: 5'-UUCUCC GAACGUGUCACGUdTdT-3' (QIAGEN Germany).

\section{STEREOTAXIC SURGERY AND SIRNA ADMINISTRATION IN RAT BRAIN}

Surgical procedures were carried out as described previously (13). Briefly, after general anesthesia, guide cannula was inserted into D3V (anteroposterior: $-0.5 \mathrm{~mm}$ relative to bregma, mediolateral: $0 \mathrm{~mm}$, and dorsoventral: $-3 \mathrm{~mm}$ from the skull surface) (14).

\section{WESTERN BLOTTING}

For molecular experiments, animals were decapitated and hippocampus, prefrontal cortex, and amygdala were dissected. Then, tissues were homogenized and protein concentration was measured using Bradford method (15). Next, proteins were separated on SDS-PAGE and then transferred to Polyvinylidene fluoride membrane (Millipore, Billerica, MA, USA). Blots were blocked and then probed with specific primary antibodies against cytochrome $\mathrm{c}$ and $\beta$-actin from Cell Signaling Technology (Beverly, USA), PGC-1 $\alpha$ from ABCAM (Cambridge, UK), NRF-1 from Santa Cruz Biotechnology (Santa Cruz, CA, USA) and TFAM from BioVision (Palo Alto, CA, USA) overnight (1:1000 dilution) followed by incubation with horse radish peroxidase-conjugated secondary antibody. Immunoreactivity was visualized using the enhanced chemiluminescence method (Amersham Bioscience, Piscataway, USA). Finally, densitometric analysis of protein bands was done by Image $\mathbf{J}$ software.

\section{MEASUREMENT OF CS ACTIVITY}

Based on a method described by Craig (16) , CS activity was assayed. Briefly, protein extract was added to assay buffer containing Tris-HCl, Triton X-100, acetylCoA, 5-5'-dithiobis (2-nitrobenzoic acid) and oxaloacetate. Alterations in the absorbance were recorded at $412 \mathrm{~nm}$ wavelength.

\section{MDH ACTIVITY ASSESSMENT}

To determine the activity of MDH, protein extract was added to mixture composed of oxaloacetate, Tris- $\mathrm{HCl}$ and $\mathrm{NADH}$. Absorbance was measure at $340 \mathrm{~nm}$, spectrophotometerically (16-18).

\section{DATA ANALYSIS}

All experiments were executed (replicated) at least three times with consistent results. Data analysis was conducted by using Graphpad Prism ${ }^{\circledR}$ Software using a one-way analysis of variance (ANOVA) followed by the Tukey's test for multiple comparisons, where appropriate. The data are expressed as means \pm SEM, and the level of statistical significance was considered when P-value $<0.05$. 


\section{RESULTS}

\section{Nrf2 SILENCING LED TO DECREASE IN PROTEIN LEVEL OF UPSTREAM FACTOR PGC-1 $\alpha$ IN HIPPOCAMPUS}

As shown in Fig. 1, intraventricular injection of Nrf2-siRNA significantly decreased protein level of PGC-1 $\alpha$ compared to control rats in hippocampus region 4 and $8 \mathrm{~h}$ after injection. Despite the changes in PGC-1 $\alpha$ level, as illustrated in Fig. 1C, 1D and $1 \mathrm{E}$, mitochondrial biogenesis downstream molecules NRF-1, TFAM and cytochrome c did not significantly change following Nrf2 silencing in hippocampus 4 and $8 \mathrm{~h}$ after siRNA injection.

(A)

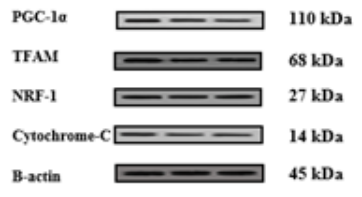

(B)

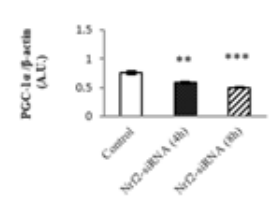

(C)

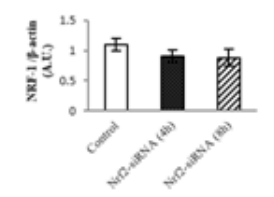

(D)

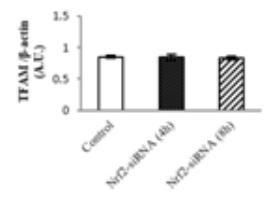

(E)

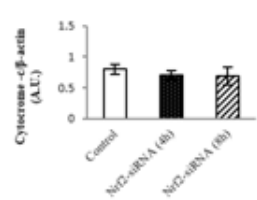

Fig.1: The effects of Nrf2 silencing on PGC-1 $\alpha$, cytochrome-c, NRF-1, and TFAM protein levels in hippocampus. A) Western blot analysis of hippocampus dissected from control and Nrf2-siRNA injected rats. Blots were probed with anti- PGC-1 $\alpha$, NRF-1, TFAM and cytochrome-c antibodies. Quantification of B) PGC-1 $\alpha$, C) NRF-1, D) TFAM and E) cytochrome $c$ in hippocampus of control and Nrf2-siRNA injected rats. Shown are mean \pm SEM. All experiment was replicated at least three times with consistent results. ${ }^{* *} \mathrm{P}<0.01 ; * * * \mathrm{P}<0.001$ compared to the control group.

PGC-1 $\alpha$ BUT NOT ITS DOWNSTREAMS SIGNIFICANTLY DECREASED AFTER Nrf2-siRNA INJECTION IN PREFRONTAL CORTEX

As illustrated in Fig. 2, Nrf2-siRNA injection decreased PGC-1 $\alpha$ protein level in prefrontal cortex compared to the control group 4 and $8 \mathrm{~h}$ later. After $8 \mathrm{~h}$, protein level of PGC- $1 \alpha$ decreased $23 \%$ in comparison with control rats. Even though protein level of NRF-1 decreased in Nrf2-siRNA-injected rats 4 and $8 \mathrm{~h}$ after injection, these reductions were not statistically significant compared to control rats (Fig. 2C). Furthermore, as shown in Fig. 2D and 2E, protein level of TFAM and cytochrome $\mathrm{c}$ did not show any significant alteration in Nrf2-siRNA injected rats in comparison with control group 4 and $8 \mathrm{~h}$ after injection. 
Brain regions and mitochondrial biogenesis

(A)

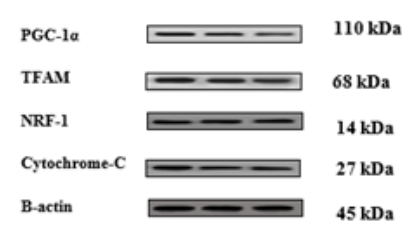

(B)

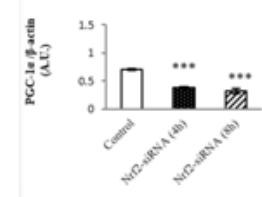

(C)

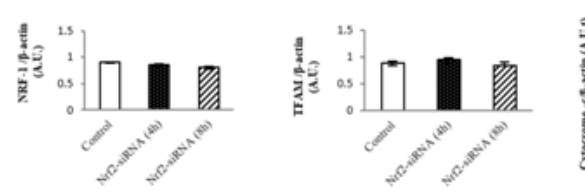

(E)

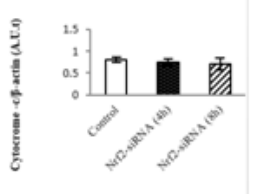

Fig.2: The effects of Nrf2 silencing on PGC-1 $\alpha$, cytochrome-c, NRF-1, and TFAM protein levels in prefrontal cortex. A) Western blot analysis of prefrontal cortex dissected from control and Nrf2-siRNA injected rats. Blots were probed with anti- PGC-1 $\alpha$, NRF-1, TFAM and cytochrome-c antibodies. Quantification of B) PGC-1 $\alpha$, C) NRF-1, D) TFAM and E) cytochrome $\mathrm{c}$ in prefrontal cortex of control and Nrf2-siRNA injected rats. Shown are mean \pm SEM. All experiment was replicated at least three times with consistent results. $* * * \mathrm{P}<0.001$ compared to the control group.

\section{INTRAVENTRICULAR INJECTION OF Nrf2-siRNA NOTABLY DECLINED PGC-1 $\alpha$ PROTEIN LEVEL IN AMYGDALA}

As the master regulator of mitochondrial biogenesis, protein level of PGC- $1 \alpha$ significantly decreased in amygdala 4 and $8 \mathrm{~h}$ after Nrf2-siRNA intraventricular injection (Fig. 3B). Like the hippocampus and prefrontal cortex, decrease in protein level of NRF-1 was not significant compared to control group 4 and $8 \mathrm{~h}$ after Nrf2 silencing (Fig. 3C). Also, protein level of TFAM and cytochrome $\mathrm{c}$ did not alter after Nrf2-siRNA injection as shown in Fig. 3D and 3E.

(A)
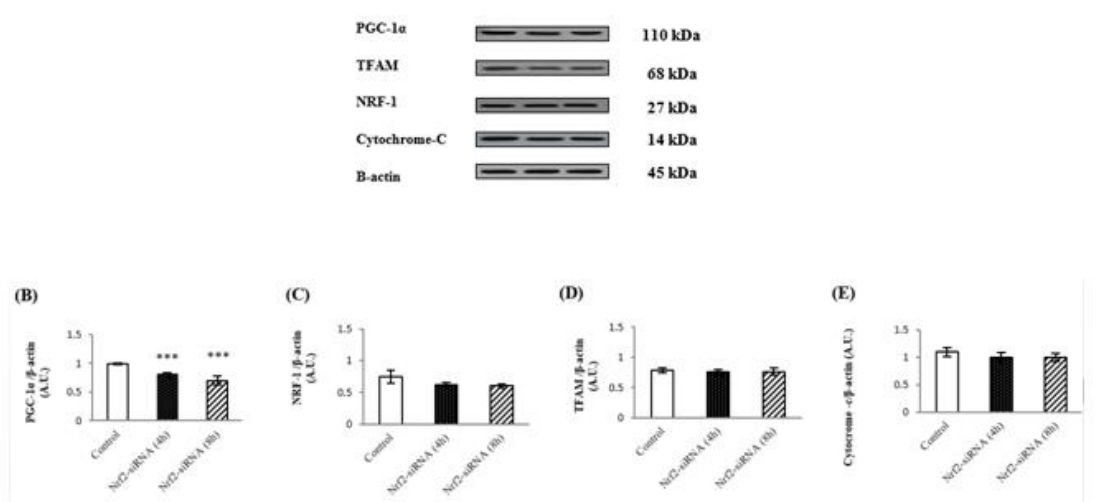

Fig.3: The effects of Nrf2 silencing on PGC-1 $\alpha$, cytochrome-c, NRF-1, and TFAM protein levels in amygdala. A) Western blot analysis of amygdala dissected from control and Nrf2-siRNA injected rats. Blots were probed with anti- PGC-1 $\alpha$, NRF-1, TFAM and cytochrome-c antibodies. Quantification of B) PGC-1 $\alpha$, C) NRF-1, D) TFAM and 
E) cytochrome c in amygdala of control and Nrf2-siRNA injected rats. Shown are mean \pm SEM. All experiment was replicated at least three times with consistent results. $* * * \mathrm{P}<0.001$ compared to the control group.

\section{Nrf2 SILENCING AFFECTED ACTIVITY OF TWO ENZYMES CS AND MDH IN HIPPOCAMPUS, PREFRONTAL CORTEX AND AMYGDALA}

TCA cycle is an intra-mitochondrial series of enzyme-catalyzed chemical reactions that forms the central part of the aerobic respiration. Our data from biochemical assay indicated that activity of CS in hippocampus significantly increased in Nrf2siRNA injected rats compared to control group (Fig. 4A). Moreover, elevation in CS activity $4 \mathrm{~h}$ after siRNA injection was more. Interestingly, activity of MDH in hippocampus decreased significantly 4 and $8 \mathrm{~h}$ after Nrf 2 silencing, as shown in Fig. 4B. Reduction in MDH activity after $8 \mathrm{~h}$ was more rather than $4 \mathrm{~h}$.

Measurement of activity of two enzymes CS and MDH in prefrontal cortex indicated that similar to hippocampus, Nrf2 knockdown caused a notably increase in CS activity. In contrary to hippocampus, increase in activity of CS in prefrontal cortex had upward trend $8 \mathrm{~h}$ after siRNA injection. While activity of MDH in this region of brain significantly decreased compared to control rats $8 \mathrm{~h}$ after siRNA injection, the activity of MDH did not show any significant change $4 \mathrm{~h}$ after $\mathrm{Nrf} 2$ silencing in comparison with control rats (Fig. 4C and 4D).

Unlike the hippocampus and prefrontal cortex, our data revealed that activity of two enzymes CS and MDH did not significantly change following Nrf2 silencing in amygdala compared to the control group 4 and $8 \mathrm{~h}$ after injection (Fig. 4E and 4F).

A)

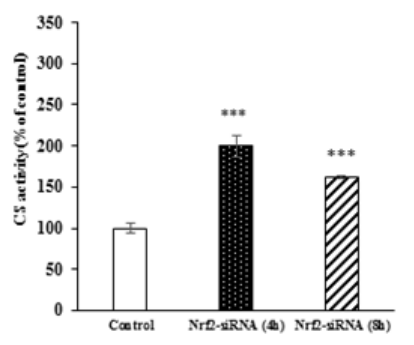

C)

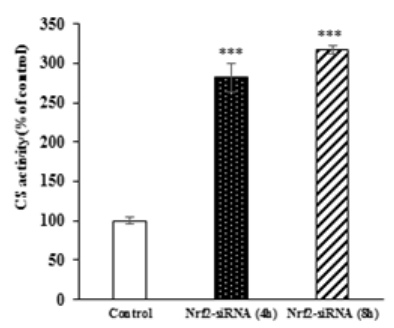

E)

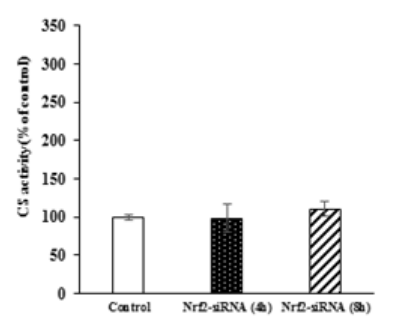

B).

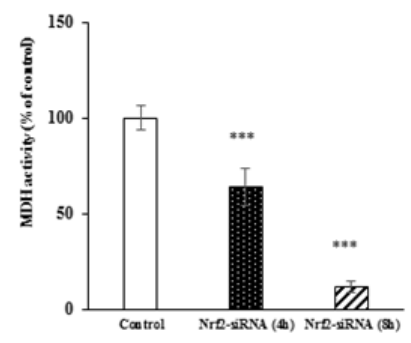

D)

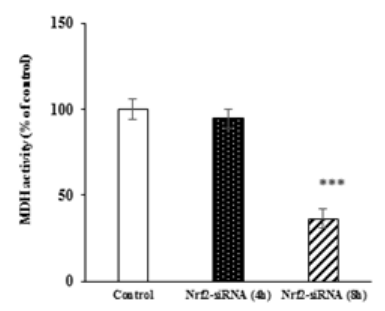

F)

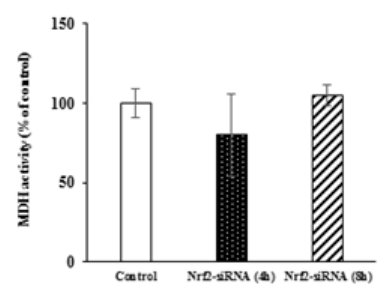

Fig.4: Effect of Nrf2 silencing on CS and MDH enzymes activities in A, B) hippocampus, C, D) prefrontal and E, F) amygdala of rat brain, respectively. $* * * \mathrm{P}<0.001$ compared to the control group. 
Brain regions and mitochondrial biogenesis

\section{DISSCUSION}

The findings presented here demonstrated that Nrf2 knockdown led to a significant reduction in PGC-1 $\alpha$ levels in three brain regions including hippocampus, amygdala and prefrontal cortex. However, there was no significant alteration in protein level of NRF-1, TFAM and cytochrome $\mathrm{c}$ in three desired regions. Moreover, these alterations were accompanied with changes in activities of specific enzymes of TCA cycle following Nrf2 knockdown. Obtained data from biochemical analysis revealed that Nrf2 knockdown increased CS activity in hippocampus and prefrontal cortex but not in amygdala. Besides, MDH activity notably decreased in hippocampus and prefrontal cortex but not in amygdala following Nrf2 knockdown.

A recent work from our laboratory has revealed that intraventricular administration of siRNA targeting Nrf2 exerted significant anxiety-like behavior in rats besides mitochondrial dysfunction and neuronal apoptosis in hippocampus, amygdala, and prefrontal cortex (18). Accordingly, the original aim of the present report was to check whether Nrf2 silencing could affect another feature of mitochondrial network quality control including mitochondrial biogenesis. Based on our findings, whilst PGC- $1 \alpha$ as a central stimulator of mitochondrial biogenesis significantly downregulated by Nrf2 knockdown, its downstream molecules including NRF-1 and TFAM was not notably influenced by Nrf2 silencing. One of the main reasons for this reduction in PGC-1 $\alpha$ protein level could be attributed to the crucial role of Nrf2 in PGC-1 $\alpha$ activation. As hypothesized by Clark and Simon, there are Nrf2 consensus sequences in PGC-1 $\alpha$ promoter that makes a redox regulatory loop between PGC-1 $\alpha$ and Nrf2 expression (19). In this line, it has been shown that first 2 $\mathrm{Kb}$ of PGC- $1 \alpha$ promoter has two ARE core sequences (20). Moreover, Adamovich et al. (21) have reported that $\mathrm{NAD}(\mathrm{P}) \mathrm{H}$ dehydrogenase [quinone] 1 (NQO1) which is a Nrf2-dependent downstream, has leading role in regulation of PGC-1 $\alpha$ activity by means of protecting PGC- $1 \alpha$ from proteasomal degradation in NADH-dependent manner. In this regard, we have reported previously (13) that as a compensatory response to Nrf1 silencing, protein level of Nrf2 increased in three regions hippocampus, amygdala and prefrontal cortex which was accompanied by elevated level of PGC-1 $\alpha$ following intraventricular injection of Nrf1-siRNA. It seems that due to the Nrf2 response sequences in PGC-1 $\alpha$ promoter, Nrf2 knockdown leads to downregulation of PGC- $1 \alpha$.

Neurons are the most highly glucose consuming cells to maintain their intrinsic functions (22). According to the importance of energy metabolism in neuronal cells, TCA cycle which include eight crucial enzymes could reflect the cerebral metabolism rate. In fact, TCA cycle as the core part of the aerobic respiration in cells includes a series of enzyme-catalyzed chemical reactions starting from CS and ending to MDH enzymes (23). Based on the main role of TCA cycle in glucose metabolism, we examined the activity of CS and MDH enzymes of the cycle following Nrf2 knockdown. Our data from biochemical analysis indicated that activity of CS increased in prefrontal cortex and hippocampus, while MDH enzyme activity decreased in those regions following Nrf2 silencing. The exact mechanism underling Nrf2 knockdown exerts its effects on TCA cycle enzymes activity remain unknown. However, it has been documented that Nrf2 participates in anabolic pathways of glucose and glutamine metabolisms(22-26). Hence, it could be supposed that $\mathrm{Nrf} 2$ silencing leads to intensify intracellular catabolism pathways ultimately, resulted in elevated level of acetyl CoA. Acetyl CoA is one of the main substrates for CS activity (25). Furthermore, Wright et al. (26) have reported that PGC-1 $\alpha$ may mediate activity of CS enzyme. Finally, our findings indicated that 
MDH activity reduced following Nrf2 silencing. In addition to regulatory effect of Nrf2 on MDH expression (26-28), it has been shown that PGC-1 $\alpha$ overexpression leads to upregulation of TCA cycle enzymes activity and expression (27-29). Considering the significant decrease in protein level of PGC-1 $\alpha$ in Nrf2 siRNA injected rats, it is expected that decrease in activity of $\mathrm{MDH}$ is due to downregulation of PGC-1 $\alpha$ alongside Nrf2 knockdown $(29,30)$. Although the exact mechanism that Nrf2 exerts its effects on mitochondrial energy homeostasis remain unknown, it is obvious that there is a cross-talk between Nrf2 and various intracellular metabolic systems. Further studies are required to clarify the direct link between Nrf2 and mitochondrial function.

\section{CONCLUSION}

In conclusion, the present findings highlighted complex interaction of Nrf2 with mitochondrial functions in a brain region-specific manner. The results of the present study suggested a specific role of Nrf2 in modulation of mitochondrial biogenesis in brain. Moreover, our data revealed that Nrf2 knockdown has contradictory effects on TCA cycle enzymes activity in hippocampus, prefrontal cortex and amygdala. However, by outlining the exact interaction between Nrf2 as the key regulator of antioxidant defense system and mitochondria as the main intracellular sources of ROS, it would be possible to find new therapeutic strategies for neurological disorders related to oxidative stress.

\section{REFERENCES}

1. Duchen MR. Roles of mitochondria in health and disease. Diabetes. 2004;53 Suppl 1:S96-102.

2. Palikaras K, Tavernarakis N. Mitochondrial homeostasis: The interplay between mitophagy and mitochondrial biogenesis. Experimental Gerontology. 2014;56:182-8.

3. Zhu J, Wang KZQ, Chu CT. After the banquet. Autophagy. 2013;9(11):1663-76.

4. Jornayvaz FR, Shulman GI. Regulation of mitochondrial biogenesis. Essays in biochemistry. 2010;47:69-84.

5. Scarpulla RC, Vega RB, Kelly DP. Transcriptional integration of mitochondrial biogenesis. Trends in Endocrinology \& Metabolism. 2012;23(9):459-66.

6. Scarpulla RC. Metabolic control of mitochondrial biogenesis through the PGC-1 family regulatory network. Biochimica et Biophysica Acta (BBA) - Molecular Cell Research. 2011;1813(7):1269-78.

7. Wu Z, Puigserver P, Andersson U, Zhang C, Adelmant G, Mootha V, et al. Mechanisms controlling mitochondrial biogenesis and respiration through the thermogenic coactivator PGC-1. Cell. 1999;98(1):115-24.

8. Zhang L, Zhou R, Li X, Ursano RJ, Li H. Stress-induced change of mitochondria membrane potential regulated by genomic and non-genomic GR signaling: A possible mechanism for hippocampus atrophy in PTSD. Medical Hypotheses. 2006;66(6):1205-8.

9. Itoh K, Tong KI, Yamamoto M. Molecular mechanism activating Nrf2-Keap1 pathway in regulation of adaptive response to electrophiles. Free Radical Biology and Medicine. 2004;36(10):1208-13.

10. Biswas M, Kwong EK, Park E, Nagra P, Chan JY. Glycogen synthase kinase 3 regulates expression of nuclear factor-erythroid-2 related transcription factor-1 (Nrf1) and inhibits pro-survival function of Nrf1. Experimental Cell Research. 2013;319(13):1922-31.

11. Li P, Zhao Q-L, Wu L-H, Jawaid P, Jiao Y-F, Kadowaki M, et al. Isofraxidin, a potent reactive oxygen species (ROS) scavenger, protects human leukemia cells from radiationinduced apoptosis via ROS/mitochondria pathway in p53-independent manner. Apoptosis. 2014;19(6):1043-53.

12. Zhang M, An C, Gao Y, Leak RK, Chen J, Zhang F. Emerging roles of Nrf2 and phase II antioxidant enzymes in neuroprotection. Progress in neurobiology. 2013;100:30-47. 
Brain regions and mitochondrial biogenesis

13. Khalifeh S, Oryan S, Khodagholi F, Digaleh H, Shaerzadeh F, Maghsoudi N, et al. Complexity of Compensatory Effects in Nrf1 Knockdown: Linking Undeveloped Anxiety-Like Behavior to Prevented Mitochondrial Dysfunction and Oxidative Stress. Cellular and molecular neurobiology. 2015:1-11.

14. G Paxinos CW. The rat brain in stereotaxic coordinates. Elsevier Academic press, San Diego. 2007.

15. Bradford MM. A rapid and sensitive method for the quantitation of microgram quantities of protein utilizing the principle of protein-dye binding. Analytical Biochemistry. $1976 ; 72(1): 248-54$.

16. Craig I. A procedure for the analysis of citrate synthase (E. C 4137) in somatic cell hybrids. Biochemical Genetics. 1973; 9: $351-358$.

17. Mullinax TR, Mock J, McEvily A, Harrison J. Regulation of mitochondrial malate dehydrogenase. Evidence for an allosteric citrate-binding site. Journal of Biological Chemistry. 1982;257(22):13233-9.

18. Khalifeh S, Oryan S, Digaleh H, Shaerzadeh F, Khodagholi F, Maghsoudi N, et al. Involvement of $\mathrm{Nrf} 2$ in development of anxiety-like behavior by linking $\mathrm{Bcl} 2$ to oxidative phosphorylation: estimation in rat hippocampus, amygdala, and prefrontal cortex. Journal of Molecular Neuroscience. 2015;55(2):492-9.

19. Clark J, Simon DK. Transcribe to survive: transcriptional control of antioxidant defense programs for neuroprotection in Parkinson's disease. Antioxidants \& redox signaling. 2009;11(3):509-28.

20. Wasserman WW, Fahl WE. Functional antioxidant responsive elements. Proceedings of the National Academy of Sciences. 1997;94(10):5361-6.

21. Adamovich Y, Shlomai A, Tsvetkov P, Umansky KB, Reuven N, Estall JL, et al. The Protein Level of PGC-1 $\alpha$, a Key Metabolic Regulator, Is Controlled by NADH-NQO1. Molecular and Cellular Biology. 2013;33(13):2603-13.

22. Bélanger M, Allaman I, Magistretti Pierre J. Brain Energy Metabolism: Focus on Astrocyte-Neuron Metabolic Cooperation. Cell Metabolism. 2011;14(6):724-38.

23. Fernie AR, Carrari F, Sweetlove LJ. Respiratory metabolism: glycolysis, the TCA cycle and mitochondrial electron transport. Current Opinion in Plant Biology. 2004;7(3):25461.

24. Hayes JD, Ashford ML. Nrf2 orchestrates fuel partitioning for cell proliferation. Cell metabolism. 2012;16(2):139-41.

25. Hayes John D, Ashford Michael LJ. Nrf2 Orchestrates Fuel Partitioning for Cell Proliferation. Cell Metabolism. 2012;16(2):139-41.

26. Wright DC, Han D-H, Garcia-Roves PM, Geiger PC, Jones TE, Holloszy JO. Exerciseinduced Mitochondrial Biogenesis Begins before the Increase in Muscle PGC-1 $\alpha$ Expression. Journal of Biological Chemistry. 2007;282(1):194-9.

27. Thimmulappa RK, Mai KH, Srisuma S, Kensler TW, Yamamoto M, Biswal S. Identification of Nrf2-regulated genes induced by the chemopreventive agent sulforaphane by oligonucleotide microarray. Cancer research. 2002;62(18):5196-203.

28. Hatazawa Y, Senoo N, Tadaishi M, Ogawa Y, Ezaki O, Kamei Y, et al. Metabolomic Analysis of the Skeletal Muscle of Mice Overexpressing PGC-1 $\alpha$. PloS one. 2015;10(6):e0129084.

29. Roselli CE, Klosterman S, Resko JA. Anatomic relationships between aromatase and androgen receptor mRNA expression in the hypothalamus and amygdala of adult male cynomolgus monkeys. The Journal of Comparative Neurology. 2001;439(2):208-23.

30. Franklin RB, Kahng M, Akuffo V, Costello L. The effect of testosterone on citrate synthesis and citrate oxidation and a proposed mechanism for regulation of net citrate production in prostate. Hormone and metabolic research $=$ Hormon-und Stoffwechselforschung= Hormones et metabolisme. 1986;18(3):177-81. 


\section{Erratum}

In Article "Brain Region Specificity of Mitochondrial Biogenesis and Bioenergetics Response to Nrf2 Knockdown: A Comparison Among Hippocampus, Prefrontal Cortex and Amygdala of Male Rat Brain", with DOI number: http://dx.doi.org/10.1590/1678-4324-2017160744, published in journal Brazilian Archives of Biology and Technology, vol. 60, the 01 page.

That read:

“http://dx.doi.org/10.190/1678-4324-2017160744”

Read:

“6ttp://dx.doi.org/10.1590/1678-4324-2017160744" 